\title{
Defect-induced change of temperature-dependent elastic constants in BCC iron
}

\author{
Ning Gao ${ }^{\mathrm{a}, *}$, Wahyu Setyawan ${ }^{\mathrm{b}}$, Zhiguang Wang ${ }^{\mathrm{a}}$ \\ ${ }^{a}$ Institute of Modern Physics, Chinese Academy of Science, 73000 LanZhou, PR China \\ ${ }^{b}$ Pacific Northwest National Laboratory, Richland, WA 99352, USA
}

\begin{abstract}
The effects of radiation-induced defects (randomly distributed vacancies, voids, and interstitial dislocation loops) on temperature-dependent elastic constants, $C_{11}, C_{12}$, and $C_{44}$ in BCC iron, are studied with molecular dynamics method. The elastic constants are found to decrease with increasing temperatures for all cases containing different defects. The presence of vacancies, voids, or interstitial loops further decreases the elastic constants. For a given number of point defects, distributed vacancies show the strongest effect compared to voids or interstitial loops. In addition, the elastic constant $C=C_{44}-\left(C_{11}-C_{12}\right) / 2$ has also been calculated and used to distinguish the effect of a $\langle 100\rangle$ loop from a $1 / 2\langle 111\rangle$ loop. All these results can be combined with surface acoustic wave spectroscopy method for further understanding of radiation damage.

Keywords: elastic constants, vacancy, void, dislocation loop, molecular dynamics (MD)
\end{abstract}

\section{Introduction}

Formation of supersaturated point defects (self-interstitial atoms and vacancies) through the interaction between high-energy particles and atoms in materials has been recognized to affect the properties of materials in nuclear reactors, e.g. embrittlement, hardening, swelling, irradiation creep, and so on, resulting

\footnotetext{
* Corresponding author

Email address: ning.gao@impcas.ac.cn, Tel:+869314969639 (Ning Gao)
}

Preprint submitted to Elsevier

December 2, 2016

(C) 2016. This manuscript version is made available under the Elsevier user license http://www.elsevier.com/open-access/userlicense/1.0/ 
in radiation damage [1, 2, 3, 4, 5, 6, 7, 8, 9, 10, 11]. In experiments, transmission electron microscope is usually used to characterize the evolution of microstructure [1, 4, 6, 8, 9, 10. The stress versus strain $(\sigma$ vs. $\epsilon)$ curve [12, charpy impact test [13, nanoindentation [14, and irradiation creep test [8] before and after radiation are the main measurements to estimate the radiation-induced mechanical property change. However, these experiments are all destructive methods. Historically, measurement of the change of elastic constants has been used to interpret radiation damage [15, 16, which is considered to provide more information than the measurement of electrical resistivity change [15]. Recently, measurement of elastic modulus with surface acoustic wave spectroscopy has been used again in combination with density functional theory (DFT) calculations in W-Re alloy [17] to understand swelling. It should be noted that this experimental method is non-destructive and may be extensively used in future by combining with simulation results. However, the relationship between radiation damage and elastic modulus change (EMC) should be established in advance for its further application.

Because of the structural complexity induced by radiation damage (e.g. supersaturated interstitials, vacancies, voids, and dislocation loops) and the effect of dislocation pinning [18, simulations are needed to complement the exper25 iments to understand the effect of each defect type on the EMC. With the development of simulation methods, the properties of radiation-induced defects have been extensively studied theoretically. For examples, the formation and migration energies of defects and the interaction energies between defects have been calculated for iron-based materials [19, 20, 21, 22, 23, 24. In BCC iron, the formation energy of a vacancy (an interstitial) is 1.71 (3.59) eV calculated with molecular dynamics (MD) [25], and 2.07 (3.64) eV calculated with DFT [26]), while the migration energy of a vacancy is $0.63 \mathrm{eV}$ with $\mathrm{MD}$ [25] and 0.67 $\mathrm{eV}$ with DFT [26, and the migration energy of an interstitial is $0.34 \mathrm{eV}$ with DFT [26]. The formation of interstitial dislocation loops is also reported to 35 affect the gliding of dislocations 22 , 23. Thus, the shifts of mechanical properties (e.g. radiation hardening and creep), are expected to be affected by the 
distributions of defect density and size, especially the extended defects (voids and dislocation loops). However, the possible relationship between EMC and the density and size of these defects has not been fully studied. Thus, it is nec-

40 essary and important to establish such relationship in order to use the surface acoustic wave spectroscopy and other similar experimental methods to provide more understanding of radiation damage. In this paper, the effects of vacancies, voids, and interstitial dislocation loops on elastic constant change (ECC) at different temperatures in BCC iron are studied.

\section{2. Simulation Method}

There are mainly three methods to calculate the elastic constants of a system containing various defects: (1) with the method developed by Daw and Baskes [27]; (2) through the calculation of strain energy for various strained states as suggested by Ackland [28], and (3) by the method developed by Ray et al [29]. In the first method, only the contribution from static relaxation is considered while in the second method, the temperature fluctuation is included. We follow the third method which will be described below. In addition, due to the complexity of defect type, size, and density, we employed MD method rather than DFT to explore the radiation-induced ECC. The empirical Ackland-2004 Fe potential 55 25] is used in the present work. The computational box is cubic with the $\mathrm{X}$, $\mathrm{Y}$, and $\mathrm{Z}$ axes oriented along the [100], [010], and [001] directions, respectively. Periodic boundary conditions are applied along these three directions. There are 20 unit cells along each direction, i.e. 16000 atoms in a perfect matrix. The defects are then introduced into the matrix by inserting or removing a given number of atoms accordingly. The elastic constant tensor, $C_{i j k l}$, calculated with the method developed by Ray et al [29] is given as:

$$
\begin{aligned}
C_{i j k l} \quad & =-\frac{V_{0}}{k_{B} T} \delta\left(P_{i j} P_{k l}\right) \\
& +\frac{2 N k_{B} T}{V_{0}}\left(\delta_{i l} \delta_{j k}+\delta_{i k} \delta_{j l}\right) \\
& \left.+\frac{1}{V_{0}}<\sum_{b>a} f\left(r_{a b}\right) x_{a b i} x_{a b j} x_{a b k} x_{a b l}\right)>_{a v}
\end{aligned}
$$


where $P_{i j}$ is the stress component of $\sigma$ calculated by Parrinello-Rahman method [30] which has included the contributions from momentum and force of $N$ particles in volume $V_{0} . f$ denotes $r^{-2}\left(u^{\prime \prime}-r^{-1} u^{\prime}\right) . u$ is the potential energy and the last term in RHS of equation (1) is then equivalent to the equation (8) defined in Ref[27] by Daw and Baskes. $\bar{x}_{a b}$ is the vector connecting particles $a$ and $b$ with length $r_{a b} . k_{B}$ is Boltzman constant and $T$ is temperature. $\delta$ is defined with following equation: $\delta(\alpha, \beta)=\langle\alpha \beta\rangle_{a v}-\langle\alpha\rangle_{a v}\langle\beta\rangle_{a v}$. $\delta_{i j}$ is Kronecker delta. It should be noted that the temperature effect has also been included in this formula. In this equation, the elastic constant is a fourth-order tensor and there are three independent elastic constants in cubic crystals: $C_{11}=C_{1111}, C_{12}=C_{1122}$ and $C_{44}=C_{2323}$.

In the present work, the following defects are investigated: (1) vacancies at various concentrations, (2) a void with different sizes, and (3) $1 / 2\langle 111\rangle$ and $\langle 100\rangle$ interstitial loops with different radii. For the first case, the vacancies are introduced into the matrix randomly with a separation distance more than $7 \AA$ between vacancies. The computational boxes with the number of vacancies from 1 to 320 are calculated. Since the interstitials prefer to form dislocation loops, the effect of interstitials with different concentration is not considered here but rather the effect of interstitial prismatic loops with different radii is simulated. The number of interstitials in a loop is set from 7 to 151 for the $1 / 2\langle 111\rangle$ loop and from 37 to 161 for the $\langle 100\rangle$ loop. For systems with a void, the void is assumed to be spherical. The number of vacancies in a void embedded in the matrix is varied from 15 to 331. After the defects are introduced, the box is firstly relaxed with molecular static (MS) method. The formation energy of these defects are calculated with the following equation:

$$
E^{f}(\text { defect })=E_{\text {tot }}(\text { defect })-(N \pm m) \times E_{\text {perfect }}
$$

where $E_{\text {tot }}($ defect $)$ is the total energy of the system which contains $m$ vacancies or interstitials. $N$ is the number of atoms in a perfect matrix. Thus, $N \pm m$ 75 is the total number of atoms in the system after including defects. $E_{\text {perfect }}$ is the energy per atom in the perfect matrix. After MS relaxation, the MD 
Table 1: Elastic constants, $C_{11}, C_{12}$, and $C_{44}$, of a perfect BCC Fe at different temperatures.

\begin{tabular}{lccr}
\hline Temperature $(\mathrm{K})$ & $C_{11}(\mathrm{GPa})$ & $C_{12}(\mathrm{GPa})$ & $C_{44} \mathrm{GPa}$ \\
\hline 0 & 243.323 & 145.043 & 116.141 \\
100 & 237.940 & 138.981 & 110.222 \\
200 & 232.790 & 132.000 & 104.450 \\
300 & 225.420 & 130.530 & 99.242 \\
450 & 218.730 & 125.180 & 95.302 \\
600 & 213.449 & 119.550 & 93.020 \\
700 & 208.950 & 118.530 & 91.652 \\
\hline
\end{tabular}

simulations are performed at a given temperature $(0 \mathrm{~K}$ to $700 \mathrm{~K})$ in which the velocity scaling method is used. For all calculations, the NPT ensemble is applied with zero pressure. The time-step is $1 \mathrm{fs}$ and the total simulation time for each case is up to 100 ps. The elastic constants are then calculated after above full relaxations at different temperatures. The state obtained from NPT is then set as initial state for further 20,000 MD steps relaxation under NVT ensemble. The relaxations from the last $1000 \mathrm{MD}$ steps are used for elastic constant calculation at different temperatures with equation (1) to ensure the statistic accuracy.

\section{Results}

The elastic constants, $C_{11}, C_{12}$, and $C_{44}$, of perfect matrix at different temperatures are firstly calculated as a reference. These results are listed in Table 1. These elastic constants decrease with increasing temperatures, consistent with experimental results [31, 32]. In other materials (e.g. Si [33] and Au [34]), a similar trend is also observed. The fitted functions for the dependence of elastic 

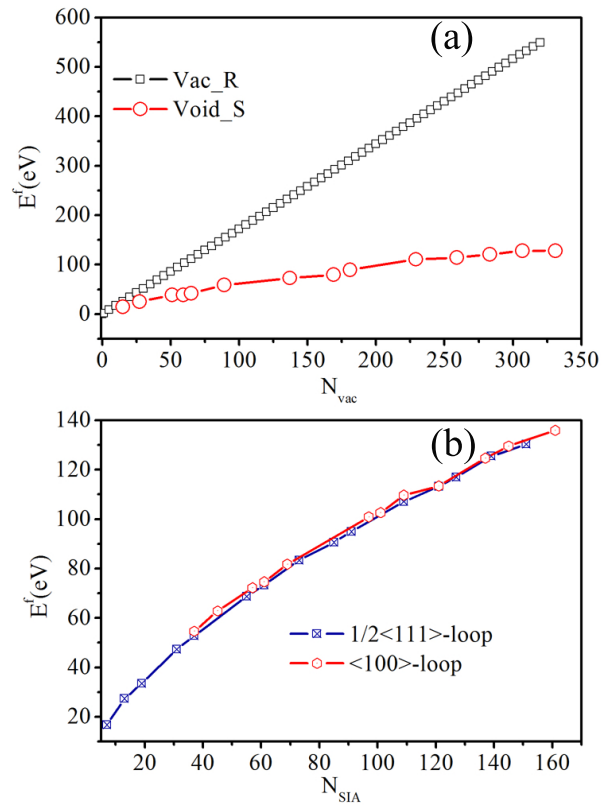

Figure 1: (a) Formation energy of randomly (R) distributed vacancies and single (S) void with number of vacancies. (b) Formation energy of $1 / 2\langle 111\rangle$ and $\langle 100\rangle$ interstitial loop with number of initerstitials.

constants on temperature are:

$$
\begin{aligned}
& C_{11}=-0.04936 T+242.0 \\
& C_{12}=3.18591 \times 10^{-5} T^{2}-0.05979 T+144.7 \\
& C_{44}=4.87617 \times 10^{-5} T^{2}-0.06903 T+116.4
\end{aligned}
$$

The standard errors of the fitted parameters in linear function are $3.0 \times 10^{-3}$ and 1.0 for slope and intercept, respectively, while in quadratic functions $\left(a x^{2}+\right.$ $b x+c)$, the maximal standard errors of $a, b$ and $c$ are $1.0 \times 10^{-5}, 3.0 \times 10^{-3}$ and 1.4, respectively. These values also cover the standard errors of the following equations which describe the dependence of elastic constants on temperatures with the effect of defects. 

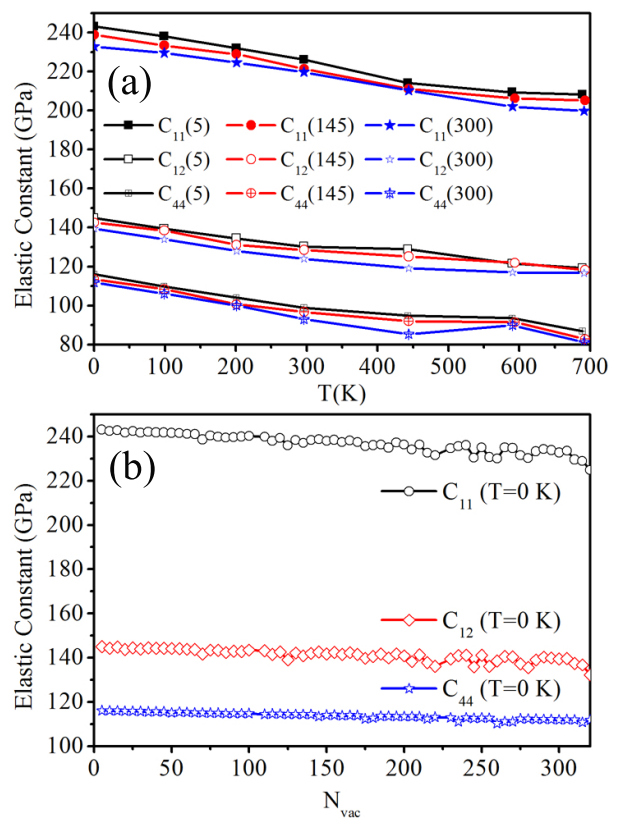

Figure 2: Elastic constants, $C_{11}, C_{12}$, and $C_{44}$, of a system containing randomly distributed vacancies (a) as a function of temperature for three cases of vacancy density (the number of vacancies in each case is given in parentheses) and (b) as a function of the number of vacancies at $0 \mathrm{~K}$.

\subsection{Effect of vacancy on elastic constants change}

After the relaxation with MS, the formation energy of $N_{v a c}$ random vacancies increases linearly as a function of $N_{v a c}$ as shown by the curve Vac_R in Figure 1(a). The dependencies of elastic constants $\left(C_{11}, C_{12}\right.$, and $\left.C_{44}\right)$ on vacancy density and temperature are shown in Figure 2, In order to show clearly, Fig 2(a) only shows three different cases of vacancy density, noting that the trend is similar for other cases. The decrease of value with increasing temperature is observed for all three elastic constants in the sample containing a different number of vacancies, as shown in Fig 2(a). Such a decrease has also been observed in a perfect matrix as shown in Table 1. With increasing temperature from 0 to $700 \mathrm{~K}, C_{11}, C_{12}$, and $C_{44}$ decrease by about $12.5 \%, 14.3 \%$, and $20.0 \%$, respectively. The fitted functions between elastic constants under 
effect of randomly distributed vacancies and temperatures are:

$$
\begin{aligned}
& C_{11}^{v a c}=-0.05336 T+239.0 \\
& C_{12}^{v a c}=3.18591 \times 10^{-5} T^{2}-0.06235 T+144.7 \\
& C_{44}^{v a c}=4.87617 \times 10^{-5} T^{2}-0.07556 T+116.4
\end{aligned}
$$

Therefore, the loss of shear resistance with temperature has a serious effect on the mechanical stability of BCC iron containing vacancies. Comparing with previous results [31, 32] and fitted equations (3) to (5), these results indicate that randomly distributed vacancies decrease the elastic constants.

One example of the detailed trend of elastic constants with increasing vacancy number calculated for the case of $0 \mathrm{~K}$ is shown in Fig 2(b). The reductions of $C_{11}, C_{12}$, and $C_{44}$ are around $4.2 \%, 5.0 \%$ and $4.3 \%$, respectively when the vacancy density reaches around $2 \%$. From these results, it can be concluded that in addition to increasing temperature, increasing vacancy density can reduce the elastic constants of BCC iron.

\subsection{Effect of void on elastic constants change}

When individual vacancies cluster together to form a spherical void, the formation energy is different from that of isolated vacancies. The results are shown in Fig1(a). Compared to the case of randomly distributed vacancies, the clustering of vacancies significantly decreases the total energy of the system. The elastic constants, $C_{11}, C_{12}$, and $C_{44}$, are then calculated for systems containing a single void of various sizes as a function of temperature from 0 to $700 \mathrm{~K}$. The results are shown in Figure 3(a). The elastic constants decrease with increasing temperatures, similar to the case of randomly distributed vacancies, as previously shown in Fig 2 (a). The linear and quadratic functions are also found to be adequate to describe the temperature dependence of the elastic constants with 

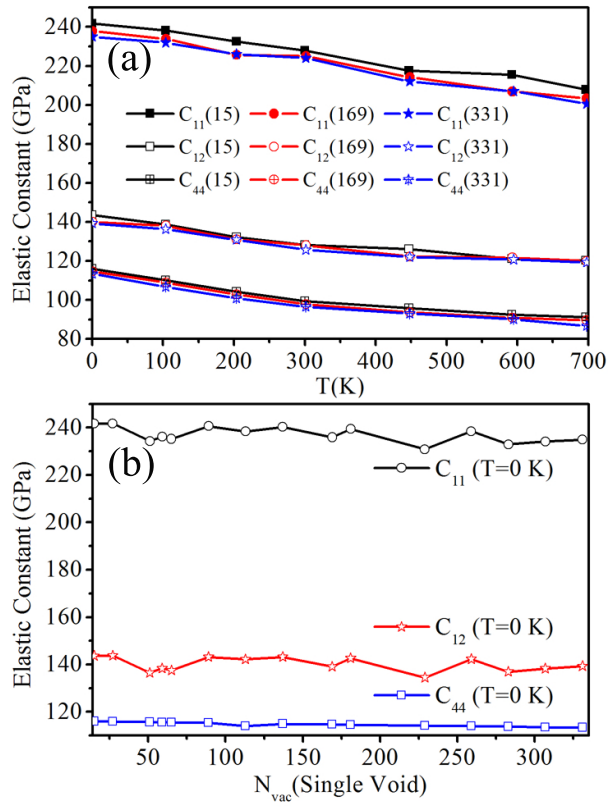

Figure 3: Elastic constants, $C_{11}, C_{12}$, and $C_{44}$, of a system containing a void (a) as a function of temperature for three cases of void size (the number of vacancies in each case is given in parentheses) and (b) as a function of void size (vacancy number) at $0 \mathrm{~K}$.

very small changes of parameters as used in equations (3) to (8):

$$
\begin{aligned}
& C_{11}^{\text {void }}=-0.05103 T+238.5 \\
& C_{12}^{\text {void }}=3.18591 \times 10^{-5} T^{2}-0.06000 T+144.7 \\
& C_{44}^{\text {void }}=4.87617 \times 10^{-5} T^{2}-0.07556 T+116.4
\end{aligned}
$$

With the increasing number of vacancies in the void, the elastic constants are found to decrease only very slightly, as shown in Fig.3(b), which shows the results calculated at $0 \mathrm{~K}$. In fact, the trend of the elastic constants as a function of void size is similar for all temperatures. The maximum decrease is around $2 \%$ for these three elastic constants, compared to more than $4 \%$ for the case of randomly distributed vacancies (Fig 2(b)). Therefore, based on above results, the effect of randomly distributed vacancies and voids on elastic constants may be distinguished by measuring experimentally the dependence of 
elastic constants on damage dose, dpa (displacement per atom): if the elastic constants are almost constant with dpa, the vacancies exist mostly as voids rather than individual vacancies, and vice versa.
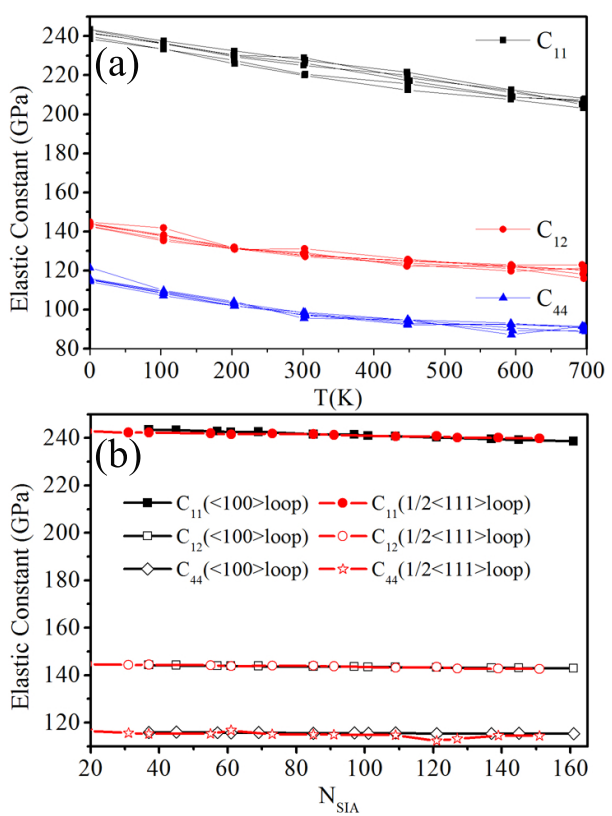

Figure 4: Elastic constants, $C_{11}, C_{12}$, and $C_{44}$, of a system containing a $1 / 2\langle 111\rangle$ loop or $\langle 100\rangle$ loop (a) as a function of temperature and (b) as a function of the loop size (number of interstitials) at $0 \mathrm{~K}$. In (a), the size of loop is not explicitly shown because the curves for both loops are indistinguishable within the statistical limit. All curves together show the trend of elastic constants as a function temperature.

\subsection{Effect of interstitial dislocation loop on elastic constants change}

The formation energies of $1 / 2\langle 111\rangle$ and $\langle 100\rangle$ loops containing different number of interstitials are shown in Fig.1(b). With same number of interstitials, the $1 / 2\langle 111\rangle$ loop has a lower formation energy than $\langle 100\rangle$ loop, indicating that the $1 / 2\langle 111\rangle$ loop is preferred at low temperatures. The three independent elastic constants, $C_{11}, C_{12}$, and $C_{44}$, are calculated for a system containing one $1 / 2$ $\langle 111\rangle$ loop or $\langle 100\rangle$ loop. The decreasing of elastic constants with temperature is observed, as shown in Figure 4(a), similar to the cases of vacancies and void. 
From the present results, it is found that the trend under the effect of a $1 / 2$

${ }_{125}\langle 111\rangle$ loop or $\langle 100\rangle$ loop is similar within the statistic range. Hence, the curves shown in Fig 4(a) are representatives of both loops with number of interstitials from 7 to 161. The dependence of elastic constants on temperatures under loop effect can be described by equation (9) to (11). With the increasing number of interstitial atoms in the loop, the elastic constants are almost unchanged (an example at $0 \mathrm{~K}$ is depicted in Fig, 4 (b) with other temperatures show the same behavior), which is same as the effect of void.

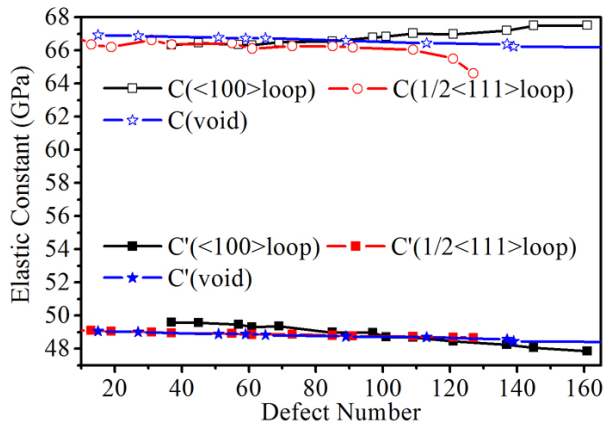

Figure 5: Elastic constants, $C^{\prime}=\left(C_{11}-C 12\right) / 2$ and $C=C_{44}-C^{\prime}$, of a system containing a $\langle 100\rangle$ loop, $1 / 2\langle 111\rangle$ loop, or void respectively as a function of defect number (interstitials in the loop or vacancies in the void).

In order to distinguish the effect of a loop from a void, the related constants $C^{\prime}=\left(C_{11}-C 12\right) / 2$ and $C=C_{44}-C^{\prime}$ are also calculated as a function of loop or void size. These results are shown in Figure $5 . C^{\prime}$ of a system containing a $\langle 100\rangle$ loop, $1 / 2\langle 111\rangle$ loop, or void decreases as a function of loop or void size, nevertheless the decrease due to the $\langle 100\rangle$ loop is larger than the other two cases. For the $C$ curves, it is interesting to note that value of $C$ increases with the number of interstitials for the system containing a $\langle 100\rangle$ loop but decreases for the system containing a $1 / 2\langle 100\rangle$ loop. For the void case, $C$ decreases slightly with the number of vacancies in the void. Combining with the results obtained in section 3.1 and 3.2, the effect on elastic constants from different defects can be then distinguished, which provides one possible way to combine with the measurement of elastic modulus with the surface acoustic wave spectroscopy 
method.

The temperature-dependent elastic constants under the effect of radiationinduced defects have been calculated with molecular dynamics method. The elastic constants, $C_{11}, C_{12}$, and $C_{44}$, in general decrease as a function of increasing temperature regardless of the type of defect in the system whether the defect is in the form of randomly distributed vacancies, voids, or interstitial dislocation loops. The elastic constants are also found to decrease as a function of the content of those defects. Furthermore, the strength of the effect among different defect types are not the same. For a given number of vacancies, the effect of individual vacancies is considerable larger than that of clustered vacancies, thus the effects of vacancies versus voids on elastic constants can be distinguished. A system containing a $\langle 100\rangle$ loop or a $1 / 2\langle 111\rangle$ loop shows an increase or decrease, respectively, of $C=C_{44}-C^{\prime}\left(\right.$ where $\left.C^{\prime}=\left(C_{11}-C 12\right) / 2\right)$ with the number of interstitials in the loop. This study shows that these types of calculations are useful to elucidate the effect of different types of defect on elastic constants which further complement the application of the surface acoustic wave spectroscopy method to understand radiation damage.

\section{Acknowledgements}

This research was supported by the US Department of Energy, Office of Fusion Energy Sciences, under Contract DE-AC06-76RLO 1830, and National Natural Science Foundation of China (Project Nos 11375245, 11675230 and 91426301).

\section{References}

[1] B. C. Master, Nature 200 (1963) 254.

[2] S. Zinkle, B. Singh, Journal of Nuclear Materials 351 (2006) 269 - 284. 
[3] J. Marian, B. D. Wirth, J. M. Perlado, Phys. Rev. Lett. 88 (2002) 255507.

[4] K. Arakawa, M. Hatanaka, E. Kuramoto, K. Ono, H. Mori, Phys. Rev. Lett. 96 (2006) 125506.

[5] H. Xu, R. E. Stoller, Y. N. Osetsky, D. Terentyev, Phys. Rev. Lett. 110 (2013) 265503.

175

[6] J. Chen, N. Gao, P. Jung, T. Sauvage, Journal of Nuclear Materials 441 (2013) $216-221$.

[7] S. L. Dudarev, R. Bullough, P. M. Derlet, Phys. Rev. Lett. 100 (2008) 135503.

[8] J. Chen, P. Jung, W. Hoffelner, H. Ullmaier, Acta Materialia 56 (2008) 250 $-258$.

[9] K. Arakawa, T. Amino, H. Mori, Acta Materialia 59 (2011) 141 - 145.

[10] Z. Yao, M. Jenkins, M. Hernndez-Mayoral, M. Kirk, Philosophical Magazine 90 (2010) 4623-4634.

[11] D. Terentyev, G. Monnet, P. Grigorev, Scripta Materialia 69 (2013) 578 581.

[12] B. Singh, A. Foreman, H. Trinkaus, J. Nucl. Mater. 249 (1997) 103-115.

[13] S. H. Kim, Y. W. Park, S. S. Kang, H. D. Chung, Nucl. Eng. Des. 212 (2002) 49-57.

[14] X. Liu, R. Wang, A. Ren, J. Jiang, C. Xu, P. Huang, W. Qian, Y. Wu, C. Zhang, J. Nucl. Mater. 444 (2014) 1-6.

[15] L. E. Rehn, J. Holder, A. V. Granato, R. R. Coltman, F. W. Young, Phys. Rev. B 10 (1974) 349-362.

[16] J. Holder, A. V. Granato, L. E. Rehn, Phys. Rev. B 10 (1974) 363-375. 
[17] F. Hofmann, D. Nguyen-Manh, M. Gilbert, C. Beck, J. Eliason, A. Maznev, W. Liu, D. Armstrong, K. Nelson, S. Dudarev, Acta Materialia 89 (2015) $352-363$.

[18] D. O. Thompson, T. H. Blewitt, D. K. Holmes, Journal of Applied Physics 28 (1957) 742-743.

[19] C. C. Fu, F. Willaime, Phys. Rev. B 72 (2005) 064117.

[20] F. Gao, H. Deng, H. L. Heinisch, R. J. Kurtz, J. Nucl. Mater 418 (2011) $115-120$.

[21] N. Gao, H. V. Swygenhoven, M. Victoria, J. Chen, Journal of Physics: Condensed Matter 23 (2011) 442201.

[22] D. Terentyev, P. Grammatikopoulos, D. J. Bacon, Y. N. Osetsky, Acta Mater. 56 (2008) 5034-5046.

[23] X. J. Shi, L. Dupuy, B. Devincre, D. Terentyev, L. Vincent, J. Nucl. Mater. 460 (2015) 37-43.

[24] A. V. Bakaev, D. A. Terentyev, P. Y. Grigor'ev, E. E. Zhurkin, Journal of Surface Investigation. X-ray, Synchrotron and Neutron Techniques 9 (2015) 290-299.

[25] G. J. Ackland, M. I. Mendelev, D. J. Srolovitz, S. Han, A. V. Barashev, J. Phys. Condens. Matter 16 (2004) S2629-S2642.

[26] C.-C. Fu, F. Willaime, P. Ordejón, Phys. Rev. Lett. 92 (2004) 175503.

[27] M. S. Daw, M. I. Baskes, Phys. Rev. B 29 (1984) 6443-6453.

[28] G. Ackland, Journal of Nuclear Materials 152 (1988) $53-63$.

[29] J. R. Ray, M. C. Moody, A. Rahman, Phys. Rev. B 32 (1985) 733-735.

[30] M. Parrinello, A. Rahman, Journal of Applied Physics 52 (1981) 7182-7190.

[31] J. A. Rayne, B. S. Chandrasekhar, Phys. Rev. 122 (1961) 1714-1716. 
[32] J. J. Adams, D. S. Agosta, R. G. Leisure, H. Ledbetter, Journal of Applied Physics 100 (2006) 113530.

[33] J. D. Schall, G. Gao, J. A. Harrison, Phys. Rev. B 77 (2008) 115209.

[34] S. Collard, R. McLellan, Acta Metallurgica et Materialia 39 (1991) 3143 3151. 